\title{
Pattern of Suicidal Methods Used in Minofiya, Gharbiya and Kafr Elshekh Governorates (2008-2012)
}

Dalia Abd-Elwahab Hassan*, Sherein S Ghaleb, Mohamed Abd Elghaffar Ahmed and Amr Reda Zaki

Forensic Medicine and Clinical Toxicology, Beni Suif University, Cairo, Egypt

\begin{abstract}
Suicide is an important health hazard worldwide. It is no longer a criminal offense in any developed country but continues to be so in many developing countries. We retrospectively analyzed the autopsy records of the Institute of Forensic Medicine during the five-year period between 2008 and 2012 to document the characteristics of fatalities resulting from suicide in Minofiya, Gharbiya and Kafr Elshekh governorates. In this study 55 suicidal cases (26 male, 29 female). Most of cases were between 20 and 50 years. Ingestion of insecticides (70.8\%) was the most common pattern of suicidal method used among the cases followed by chemical poisoning (16.36\%), hanging (9.9\%), jumping from height (1.8\%), and firearm (1.8\%). A mild seasonal variation was found, however, the rate of suicidal death was influenced by warm temperatures.
\end{abstract}

Keywords: Suicidal methods; Tranquilizers; Minofiya

\section{Introduction}

If aging and disease are eliminated and the world is made much safer (and/or surgical repair is vastly improved) so as to reduce the danger of death by accident, the major causes of death will be suicide and homicide. It is believed that no matter how advanced the technology, people will always have the means of killing other people (homicide) and of killing themselves (suicide). Suicide is one of the most important public health problems worldwide [1]. Pattern of suicidal deaths reveals the existing social and psychological state of mind of the people of a region. Suicidal behavior, pattern and rates differ in various populations and culture [2].

The choice of method used to commit suicide depends on availability of means, knowledge about lethal effectiveness, and victim's motivation. Globally, there has been a progressive increase in suicide rates, from 10.1 per 100,000 in 1950 to 16 per 100,000 populations in 1995 - a nearly $60 \%$ increase in 45 years [3]. Females of all races are more likely to report having had suicidal ideation and are almost twice as likely to attempt suicide. However, males of all races are almost five times as likely as females to die by suicide. The suicide rate in young people increased dramatically over the last few decades. In 1997, suicide was the third leading cause of death among 15-24 year olds. There are several possible reasons for committing suicide poverty: including unemployment and 'broken' homes. In many developing countries suicide remains a taboo subject because of political, religious and social sensitivities [4]. Therefore, prevention cannot truly start until this taboo is directly thought about and begin to deal with [5]. There is lack of research on suicide from developing countries. Without good robust information prevention programs cannot be developed [6].

\section{Material and Methods}

\section{Mortality data sources}

The survey target groups included victims of suicidal deaths in Minofiya, Gharbiya and Kafr El shekh governorates. We extracted daily counts of deaths cases. Information was collected from forensic autopsy records. There were 55 ( 26 male, 29 female) suicidal deaths cases autopsied in Institute of Forensic Medicine, Ministry of Justice, Minofiya, Gharbiya and Kafr El shekh governorates, from 2008 to 2012. Data were analyzed according to the types of suicidal methods, gender, and meteorological conditions. In every case there was a scene investigation performed by the police. All the cases were autopsied by forensic pathologists. The macroscopic post-mortem investigation was followed by a detailed histological examination in every case.

\section{Statistical analysis}

Collected data were tabulated then analyzed statistically. Results are expressed in numbers and percentage.

\section{Results and Discussion}

Climatic and seasonal triggering factors on human life have received an increasing public and social interest for centuries. Suicide may have been attributed to several risk factors as previous suicide attempts, alcohol/substance abuse, family history of suicide, isolation, cultural or religious beliefs that allow suicide, mental disorder and local epidemics of suicide. Physicians and meteorologists pay attention on the relationship between the meteorological events and their reactions on human health [7].

Distribution of suicidal methods is presented in Table 1. Chemical poisoning, hanging, gunshot, and jumping were the most frequent suicidal methods among males; however, ingestion of insecticide was detected in the highest number among females. The following intoxications were most frequent among males: Phenol, Benzodiazepines, Major tranquilizers and Clop Amin.

Seasonal fluctuation in suicide has been observed in many populations. High temperature may contribute to this. Our results show that seasonal transition may slightly contribute to the pathogenesis of suicide. In our material the most frequent methods, as intoxication and

*Corresponding author: Dalia Abd-Elwahab Hassan, Lecturer, Forensic Medicine and Clinical Toxicology, Beni Suif University, Cairo, Egypt, Tel: +0201223180900; E-mail: daliaabdelwahab@ymail.com

Received October 22, 2014; Accepted December 04, 2014; Published December 10,2014

Citation: Hassan DA, Ghaleb SS, Ahmed MAE, Zaki AR (2015) Pattern of Suicidal Methods Used in Minofiya, Gharbiya and Kafr Elshekh Governorates (2008-2012). J Forensic Res 6: 261. doi:10.4172/2157-7145.1000261

Copyright: (c) 2015 Hassan DA, et al. This is an open-access article distributed under the terms of the Creative Commons Attribution License, which permits unrestricted use, distribution, and reproduction in any medium, provided the original author and source are credited. 


\begin{tabular}{|c|c|c|c|}
\hline & Male no. & Female no. & All \\
\hline Ingestion of insecticides & 14 & 25 & 39 \\
\hline Chemical poisoning & 6 & 3 & 9 \\
\hline Hanging & 5 & 0 & 5 \\
\hline Jumping from height & 1 & 0 & 1 \\
\hline Firearm & 1 & 0 & 1 \\
\hline
\end{tabular}

Table 1: Distribution of suicidal methods among males and females.

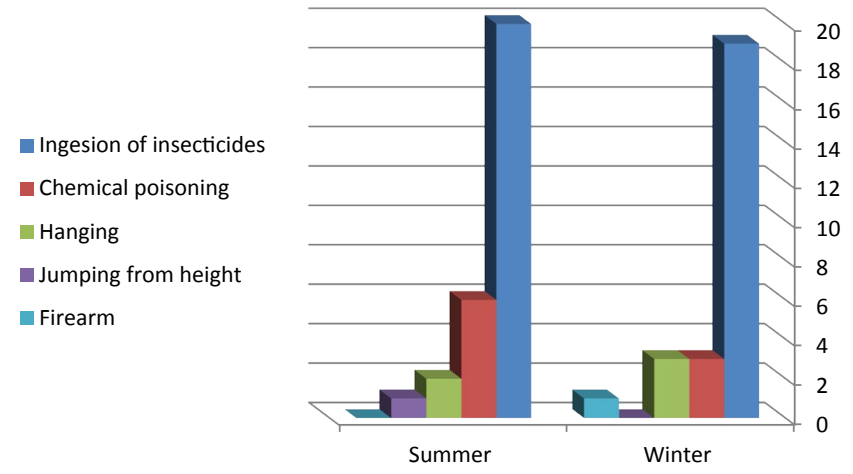

Figure 1: Seasonal distribution of different suicidal methods.

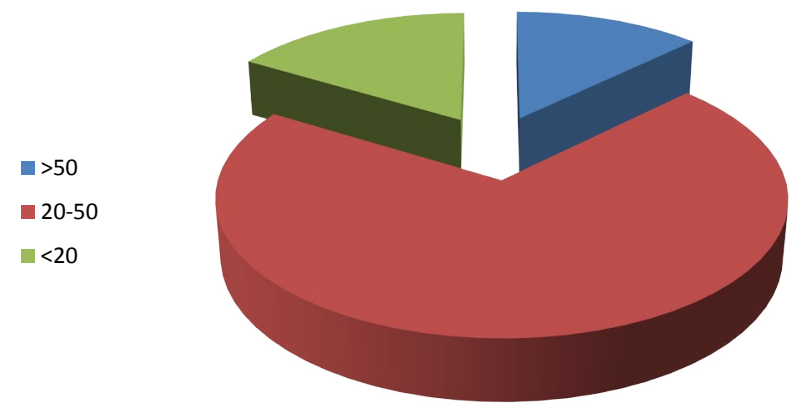

Figure 2: Age distribution between suicidal cases.

jumping from height were detected more frequently during the summer periods. The other methods did not follow seasonal distribution (Figure 1). The incidence of suicide may display a clear seasonal pattern; being positively linked with prevailing levels of sunlight [8]. Previous publications demonstrated a summer maximum and a winter minimum of suicide cases in both genders.

Mental disorders are reported frequently among the persons completing suicide [9]. It seems to be a general teaching that mental disorder occurs more frequently in autumn and winter time and not in spring [10]. However, post mortem studies suggest higher suicidal frequency in summer period than in winter time. Partonen et al. [11] found that the seasonal effect was most pronounced when the number of suicides was relatively low, and the solar and geomagnetic activity was associated with the risk of suicide. Other study has not found spring or summer peak in suicide [12].

Page [12] found an increased risk of suicide during hot weather. Above $18^{\circ} \mathrm{C}$, each $1^{\circ} \mathrm{C}$ increase in mean temperature was associated with a $3.8 \%$ and $5.0 \%$ rise in suicide and violent suicide, respectively. In contrast other results represent a novel minor effecting seasonality of suicide, which is hardly compatible with the hypothesized role of temperature in suicide seasonality. Effects of weather variables on suicide are well-documented [13]. Nevertheless, most studies show a peak in suicides during the spring season, and this is often attributed to increased temperatures. Therefore, it appears that the seasonality of suicides is due to factors other than temperature [14]. Preti [15] reported that the number of suicide cases is positively correlated to the geographical latitude. This can be explained by the fact that regions located in northern part, are less exposed to the sun and consequently the mean temperature is lower than in southern regions.

An improvement in the ability of communities to adjust to temperature changes by implementing public health interventions may play an important part in preserving the wellness of the general population, and also in limiting the worst consequences of suicidal behavior [16] The findings are of research interest for future studies regarding mechanisms of suicidal behavior, and also of practical interest for better timing of suicide interventions and effective preventive strategies. Further investigation about the characteristic injuries may help the final determination of violent death causes, and more effort should be directed to the effective prevention strategy [17].

The results of this study showed that male to female ratio was $1: 1.12$. It was observed that $87.3 \%$ of cases occurred under the age of 50 years. Peak age of suicidal attempted cases was between 20 and50 years in both males and females (Figure 2).

As regards the pattern of suicidal methods used in this study the majority of cases $70.8 \%$ were by ingesting of insecticides. $16.36 \%$ of cases attempted suicide by chemical poisoning and $9.9 \%$ of cases hanged themselves. Equal proportion $1.8 \%$ of cases attempted suicide by fire arm or jumping (intended fall) from height. Wright [1] said that the common modes of death by suicide are drowning, hanging; shooting, poisoning, jumping from heights, cutting of arteries, stabbing, and strangulation. These factors must be considered along with the ability of the deceased to accomplish the act from both a physical and a psychological stand point.

Schmidtke et al., [18] said that developed countries combined selfpoisoning ranked as the second highest suicide method for both males (14.0\%) and females (29.0\%). In the developed country this usually meant poisoning by drugs that is medication in contrast to worldwide trends which show that self-poisoning by pesticides is the frequently used method among other poisonings in many Asian, African and Latin American countries accounting in rural areas for about one-third of all suicides worldwide. In fact pesticide ingestion accounts for $58 \%$ of all suicides and accounts for $79 \%$ of all suicides among young females in China rural areas [19].

A suicide method is any means by which one or more persons purposely kill themselves. Suicide methods can be classified according to two modes of interrupting life processes physical or chemical. Physical modes of interruption typically act by in capacitating the respiratory system or the central nervous system usually by destruction of one or more key components. The availability of firearms is associated with increased suicidal ideation and the presence of a gun in the home is associated with a five times greater risk of suicide. Chemical modes focus on interrupting biologically significant processes such as cellular respiration or diffusion capacity. Chemical methods of suicide produce latent evidence of action whereas physical methods provide direct evidence [20].

This work revealed that ingestion of insecticide was the most common method of attempted suicide in both males and females. Chemical poisoning was the second method among males. There was no hanging or falling from height or fire arm case reported among females. 
In agreement with Baasher [9] who said that sex differences in the utilization of suicide methods may depend on beliefs about culturally acceptable sex-specific self-destructive behaviors. If completed suicide is viewed as a masculine behavior then an attempted suicide does not suit men's role. Following these rationale suicidal males could choose violent methods as it would make them more likely to succeed in their action. Some attribute women's use of less violent methods to their concern for bodily appearance how they will look in death. Males are significantly more likely than females to attempt suicide impulsively, whereas females are more likely to tell someone about suicidal thoughts and plans [20].

A study of suicide methods in a large number of cases in Japan and the United States revealed that Japan had a very high proportion of hanging $(70.4 \%$ for males and $60 \%$ for females). The proportion was much lower (18.2\% for males and $16.2 \%$ for females) in the United States.

Similarly an Australian study reported hanging in $32 \%$ of its cases. Hanging and self-poisoning with pesticides were the preferred means of suicide in south China. Native American males continues to be the highest of any group, whereas Caucasian males still have the highest absolute number of suicide deaths, accounting for approximately $70 \%$ of suicide. Among adolescent females, the Asian and Pacific Islander group has the highest suicide rate $[21,22]$.

In this study jumping (intended fall) from height represented $1.8 \%$ of cases. It was the 4th method of attempted suicide in males and no cases in females. In the same line it was said that jumping from height is the act of jumping from high altitudes for example from a window (selfdefenestration or auto-defenestration), balcony or roof of a high rise building, cliff, dam or bridge. In the United States jumping is among the least common methods of committing suicide (less than $2 \%$ of all reported suicides in the United States for 2005).

In contrast central Europe (Belgium, Germany and the Netherlands) and southern Europe (Italy, Portugal, and Spain including also Luxembourg) was characterized by a very high proportion of jumping [18].

In conclusion suicides in MINOFIYA, GHARBIYA AND KAFR ELSHEKH GOVERNORATES exhibit that ingestion of insecticides (70.8\%) was the most common pattern of suicidal method used among the cases and most of cases were between 20 and 50 years over the study period.

\section{References}

1. Ambade VN, Godbole HV, Kukde HG (2007) Suicidal and homicidal deaths: a comparative and circumstantial approach. J Forensic Leg Med 14: 253-260.

2. Kanchan T, Menezes RG (2008) Suicidal poisoning in Southern India: gender differences. J Forensic Leg Med 15: 7-14.

3. Kanchan T, Menezes RG (2008) Suicidal hangings in Manipal, South India victim profile and gender differences. J Forensic Leg Med 15: 493-496.

4. Meel BL (2003) A study on the incidence of suicide by hanging in the sub-region of Transkei, South Africa. J Clin Forensic Med 10: 153-157.
5. Morid M, Hanna, Etemad A, El-Shereef A, Azza H. Griew (2011) Study of pattern and outcome of suicidal methods usedin Benghazi City - Libya. Egyptian Journal of Forensic Sciences; 1: 124-132.

6. Khan MM (2005) Suicide prevention and developing countries. J R Soc Med 98: 459-463.

7. Cagle A, Hubbard R (2005) Cold-related cardiac mortality in King County Washington, USA 1980-2001. Ann Hum Biol 32: 525-537.

8. Lambert G, Reid C, Kaye D, Jennings G, Esler M (2003) Increased suicide rate in the middle-aged and its association with hours of sunlight. Am J Psychiatry 160: 793-795.

9. Zonda T, Bozsonyi K, Veres E (2005) Seasonal fluctuation of suicide in Hungary between 1970-2000. Arch Suicide Res 9: 77-85

10. Doganay Z, Sunter AT, Guz H, Ozkan A, Altintop L, et al. (2003) Climatic and diurnal variation in suicide attempts in the ED. Am J Emerg Med 21: 271-275.

11. Partonen T, Haukka J, Viilo K, Hakko H, Pirkola S, et al. (2004) Cyclic time patterns of death from suicide in northern Finland. J Affect Disord 78: 11-19.

12. Page LA, Hajat S, Kovats RS (2007) Relationship between daily suicide counts and temperature in England and Wales. Br J Psychiatry 191: 106-112.

13. Ajdacic-Gross V, Lauber C, Sansossio R, Bopp M, Eich D, et al. (2007) Seasonal associations between weather conditions and suicide--evidence against a classic hypothesis. Am J Epidemiol 165: 561-569.

14. Dixon PG, McDonald AN, Scheitlin KN, Stapleton JE, Allen JS, et al. (2007) Effects of temperature variation on suicide in five U.S. counties, 1991-2001. Int J Biometeorol 51: 395-403.

15. Dixon PG, McDonald AN, Scheitlin KN, Stapleton JE, Allen JS, et al. (2007) Effects of temperature variation on suicide in five U.S. counties, 1991-2001. Int J Biometeorol 51: 395-403.

16. Preti A (1998) The influence of climate on suicidal behaviour in Italy. Psychiatry Res 78: 9-19.

17. Preti A, Lentini G, Maugeri M (2007) Global warming possibly linked to an enhanced risk of suicide: data from Italy, 1974-2003. J Affect Disord 102: 19-25.

18. Lee HC, Lin HC, Tsai SY, Li CY, Chen CC, et al. (2006) Suicide rates and the association with climate: a population-based study. J Affect Disord 92: 221-226.

19. Schmidtke A, Weinacker B, Lo“hr C (2004) Suicide and suicide attemptsin Europe - an overview. In: Schmidtke A, Bille-Brahe U, De LeoD, et al., (eds.) Suicidal behaviour in Europe. Results from theWHO/EUROMulticentre Study on Suicidal Behaviour.Seattle,Toronto, Bern, Gottingen: Hogrete \& Huber; 1528.

20. Bertolote JM, Fleischmann A, Eddleston M, Gunnell D (2006) Deaths from pesticide poisoning: a global response. Br J Psychiatry 189: 201-203.

21. Lester D (1988) Why do people choose particular methods for suicide? Act Nerv Super (Praha) 30: 312-314.

22. Yang GH, Phillips MR, Zhou MG, Wang LJ, Zhang YP, et al. (2005) Understanding the unique characteristics of suicide in China: national psychological autopsy study. Biomed Environ Sci 18: 379-389. 